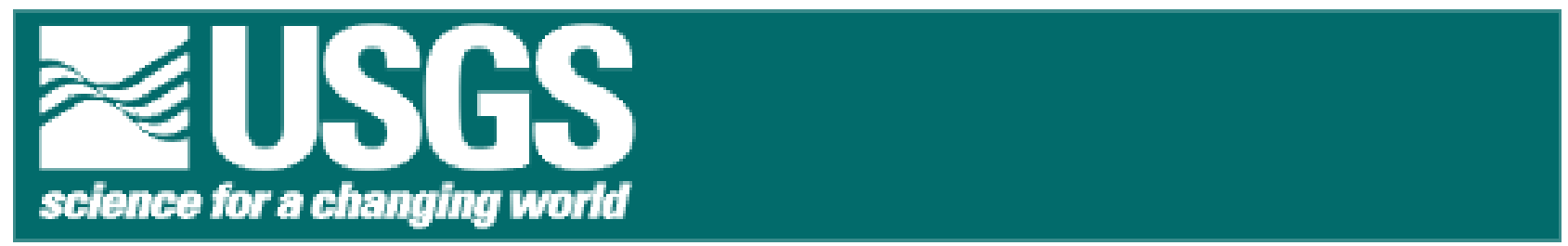

\title{
A Procedural Manual for Measurement of Uranium and Thorium Isotopes Utilizing the USGS-Stanford
}

\section{Finnegan Mat 262}

By Donald D. Shamp ${ }^{1}$

Open-File Report 01-333

2001

This report is preliminary and has not been reviewed for conformity with U.S. Geological Survey

Editorial standards. Any use of trade, firm, or product names is for descriptive purposes only and does not imply endorsement by the U.S. Government.

U.S. DEPARTMENT OF THE INTERIOR

U.S. GEOLOGICAL SURVEY

${ }^{1}$ U.S. Geological Survey, 345 Middlefield Rd., Menlo Park, CA 94025 


\section{Table of Contents}

Introduction 3

I. Filament Loading 4

II. Uranium metal......"Single Filament Method" 5

III. Thorium metal......"Single Filament Method" 12

IV. Some Miscellaneous Tips and Tricks 19

V. Figure1 4

$\begin{array}{lr}\text { Figure } 2 & 20\end{array}$

$\begin{array}{ll}\text { Figure } 3 & 21\end{array}$

$\begin{array}{lr}\text { Figure } 4 & 21\end{array}$

Figure 5 22

$\begin{array}{lr}\text { Figure } 6 & 22\end{array}$

$\begin{array}{ll}\text { Figure } 7 & 23\end{array}$

$\begin{array}{ll}\text { Figure } 8 & 23\end{array}$

$\begin{array}{lr}\text { Figure } 9 & 24\end{array}$

$\begin{array}{ll}\text { Figure } 10 & 24\end{array}$

$\begin{array}{lr}\text { Figure } 11 & 25\end{array}$ 


\title{
A Procedural Manual for Measurement of Uranium and Thorium
}

\author{
Isotopes
}

\section{Utilizing the USGS-Stanford Finnegan-Mat 262}

\section{Introduction}

Over the past several decades investigators have extensively examined the ${ }^{238} \mathrm{U}_{-}{ }^{234} \mathrm{U}-$

${ }^{230} \mathrm{Th}$ systematics of a variety of geologic materials using alpha spectroscopy.

Analytical uncertainty for ${ }^{230}$ Th by alpha spectroscopy has been limited to about $2 \%(2 \sigma)$.

The advantage of thermal ionization mass spectroscopy (TIMS), introduced by

Edwards and co-workers in the late 1980's is the increased detectability of these

isotopes by a factor of $\sim 200$, and decreases in the uncertainty for ${ }^{230}$ Th to about $5 \%$ o $(2 \sigma)$ error.

This report is a procedural manual for using the USGS-Stanford Finnegan-Mat 262 TIMS to collect and isolate Uranium and Thorium isotopic ratio data. Chemical separation of Uranium and Thorium from the sample media is accomplished using acid dissolution and then processed using anion exchange resins.

The Finnegan-Mat262 Thermal Ionization Mass Spectrometer (TIMS) utilizes a surface ionization technique in which nitrates of Uranium and Thorium are placed on a source filament. Upon heating, 
positive ion emission occurs. The ions are then accelerated and focused into a beam which passes through a curved magnetic field dispersing the ions by mass. Faraday cups and/or an ion counter capture the ions and allow for quantitative analysis of the various isotopes.

\section{FILAMENT LOADING PROCEDURES}

\section{URANIUM:}

1. Apply a $\sim 2.5 \mu$ l layer of colloidal graphite (aqua dag) to the center third of the filament and take to dryness using $\sim 1.5$ amps of current.

2. Apply the sample over the dried graphite and take to dryness with $\sim 1.8$ amps of current.

\section{THORIUM:}

1. Apply a $\sim 1.5 \mu \mathrm{l}$ layer of colloidal graphite (aqua dag) to the center third of the filament and take to dryness using $\sim 1.5$ amps of current.

2. Mix the sample with an equal amount of graphite and apply in $\sim 1 \mu l$ layers and take to dryness with $\sim 1.1$ amps of current.

3. Apply a separate $\sim 1.5 \mu \mathrm{l}$ layer of colloidal graphite (aqua dag) over the sample and take each to dryness between applications using $~ 1.5$ amps of current.

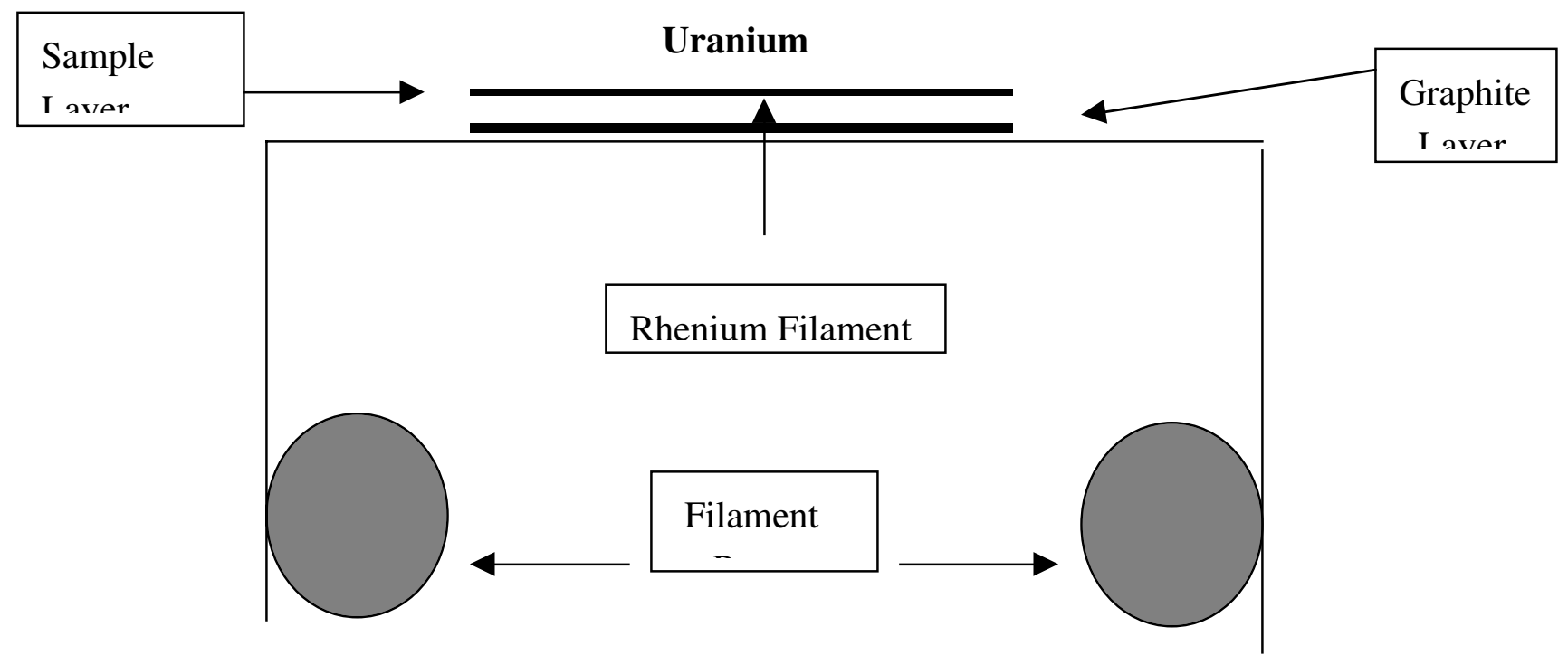




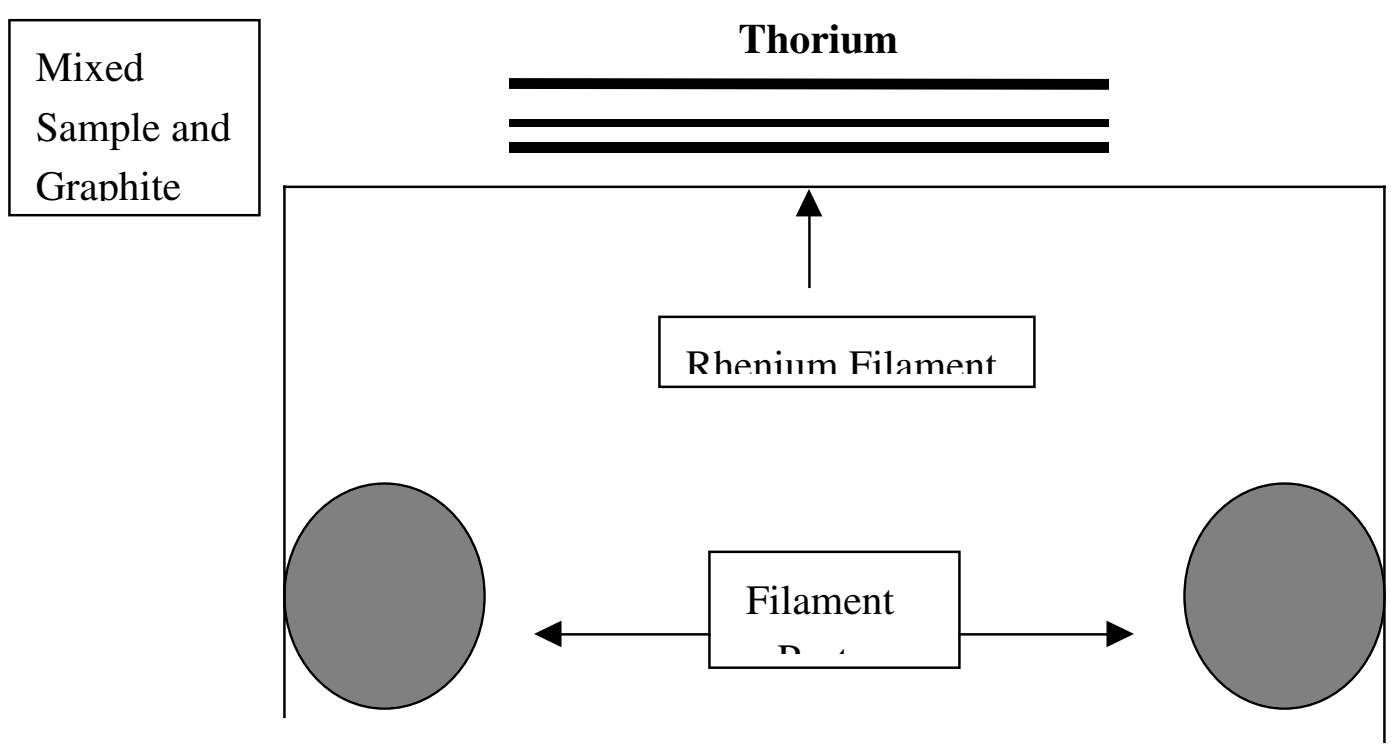

Graphite

I aver

Figure 1

$1 \mathrm{~mm}$

TIMS procedure for Uranium metal...."Single Filament Method"

\section{Start up AM:}

a. Add liquid nitrogen to carousel. Confirm that vacuum is at least $10^{-8}$ mbar.

b. Turn on "ion-source" by pushing button (figure 2). Turn high voltage (KV) from 4 to 10 (figure 3). Determine if multimeter display shows 9.8 -10 KV(figure 4). If it doesn't, press "Return to Main Keys", press "MS Control", press "Manual Mode", it will then read "Computer 
Mode", then press "Computer Mode" again. Verify that high voltage is now $\sim 9.6-10 \mathrm{KV}$.

c. Begin filament preheating when new samples have been loaded otherwise proceed to Section 3.

2. Begin filament preheating (this ensures the samples are outgassed and any organics and interfering elements are evaporated)

a. Turn KV selection knob to lowest setting (figure 3) and verify that the beam valve is closed.(figure 5).

b. Crank sample \#1 into "A” position. (figure 6)

c. Set switch on current control box to position "5". (figure 7)

d. Turn current to 3.50 amps and leave for 10 minutes.

e. Turn off current by simultaneously depressing both High Speed buttons on the filament current box.

f. Repeat steps b-e for all samples on carousel.

\section{Set Cup Configuration}

a. Press key "Return to Main keys".

b. Press key "MS Setup".

c. Press key "Cup Configuration".

d. Select Th.

e. Press key "Save Conftab". 
4. Calibration Procedure (Ensures that short term changes in the high voltage are corrected for by adjusting the magnetic field to minimize system drift across the mass range)

a. Insure calibration sample (ie; U-500) is in "IS" position \#1 (figure 6).

b. Open beam valve (figure 5).

c. Turn high voltage (KV) from 4 to 10 (figure 3).

d. Press key "Return Main Keys".

e. Press key "MS Control".

f. Press key "Input Channel”.

g. Press the number "1" key (this is the ICM counter) and press enter..

h. Press key "Input Mass".

i. Type in " 238 " and press enter.

j. Begin filament heating of U-500 sample.

k. Set switch on current control box to position "1". (figure 7)

1. Turn up current to 3.500 amps. Note the counts for " 238 ".

$\mathrm{m}$. When the count rate is several hundred, focus the beam. (see section 6 steps a-k)

n. After getting a good focus and strong count rate (several thousand cps), press key "Input Mass".

o. Type in " 235 " then do a peak center and note the count rate. It should be virtually identical to the last " 238 " count (U-500 is a 1:1 ratio of 
238/235). If it is, then the machine is counting correctly and you may proceed to calibrating the DAC table.

p. Press key "Input Mass".

q. Type in " 238 " and enter

r. Press key "Input Channel”.

s. Press key "6" (this places the 238 in the \#6 Faraday cup).

t. Press key "Return Main Keys"

u. Press key "MS Calib".

v. Press key "Magnet Calib".

w. Press key “Calib RefMass".

x. Type "238" and enter (Screen will then indicate the current ref mass in faraday \# 6).

y. Press the upper far left button on the Ops. Control panel (figures $3 \& 8$ ).

z. Turn on the chart recorder.

aa. Adjust the three magnet dials till the digital display reads in the " 238 " range and then fine tune for the strongest signal on the chart recorder (peaking to the left).

bb. Hit the space bar. The control will now shift back to the computer and the software will adjust the DAC tables and printout the results. All values should be close to 0.0 and must not exceed \pm 8.0 (figure 9). (If values exceed \pm 8.0 repeat steps $w$ through bb using 235 and 238 alternately and recheck till values are satisfactory). 
5. Begin filament heating(Ensure all samples have been pre-heated, the first sample to be analyzed is in the "IS" position and the beam valve is closed...figures $5 \& 6$ ).

a. Press key "Return to Main Keys".

b. Press key "Support Programs".

c. Press key "Heat/Ion/Eva" .

d. Press key "Autoheat Eva".

e. Type " 3500,200 " and hit enter. Ensure current is increasing on filament control box . This will take 17.5 minutes.

g. When auto-heating is complete, open the beam valve and press "Return Main Keys" (figure 5).

i. Press key "Support Programs".

j. Press key "Mass Scan", (a warning will come on screen....ignore it and press any key) .

k. Press key "Single Icm Scan".

1. Input mass limits of 232.5(low) and 238.5(high). Set Increment mass to 1.0, Integration time to 1.0, and set Max value for Y-axis to 1000 . Press enter.

m. Press key "end edit" to begin scan.

n. If 238 peak is present, slowly increase filament current till peak is at least a few hundred cps. 
o. Press key :Exit Program”.

p. Press key "Return Main Keys".

q. Press key "MS Control".

r. Press key "Input Channel”.

s. Press the number " 1 " key and enter (this is the ICM counter).

t. Press key "Input Mass".

u. Type in " 238 " and hit enter.

v. Note the present count rate.

w. Press "Peak Center" (after the machine centers the beam, the printer will register a peak center value. This value should be $512 \pm$. 8.0) and repeat if necessary to obtain a good center (figure 10).

\section{Focusing the Beam}

a. Turn on the graphing printer and remove the pen cover.

b. The printer will register the counts of the current input mass.

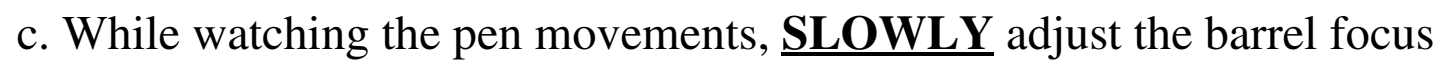
(the knob you used to put the sample filament into position...figure 6) in the direction necessary to cause the pen to move to the left (increasing). NOTE: Do not crank the knob so much as to move the filament off the electrical connection (as indicated by the sudden disappearance of current on the filament control box).....this causes the filament to quickly begin to cool down and when you return the current 
by correcting your error, the sudden zap of current may cause the sample to "pop" off the filament.

d. After getting the best count rate from the barrel focus, adjust the "shielding plate" focus knob. (figure 11)

e. Adjust the "z-deflection plate" focus (figure 11)

f. Adjust the "lens focus plates" (figure 11)

g. Press key "Peak center" (after the machine centers the beam, the printer will register a peak center value. This value should be $512 \pm .8 .0)$ and repeat if necessary to obtain a good center (figure 10).

h. You should now see a marked increase in the ion count rate on the screen.

i. Repeats steps $6 \mathrm{c}$. through $6 \mathrm{~g}$ to obtain optimum focus.

j. Turn off graphing printer and place cap back on pen tip.

k. Press key "Return Main Keys".

1. Press key "Support Programs".

m. Press key "Mass scan". (A warning will come on screen....ignore it and press any key)

n. Press key "Single Icm scan".

o. Input masses (ie. 232.5 and 238.5).

p. Press key "End Edit" to begin scan (to repeat scan...press "New Scan" and repeat steps n-p). 


\section{Optimize counting conditions.}

a. Slowly increase EVA filament current. At this point you should be in the neighborhood of around $3.500-4.300$ amps.

b. Repeat Mass scan several times and note if all peaks are present. (ie, 233, 234 and 235)

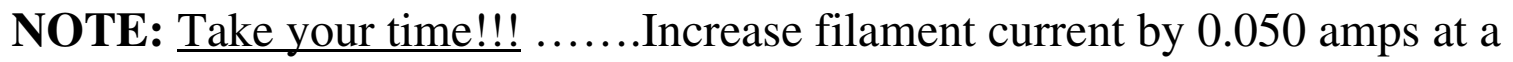
time and run mass scans several times before continuing raise the current. The trick is to SNEAK up on the correct current so as to ensure that you do not burn the sample off the filament. Ideal filament current can be as little as 3.500 amps all the way to 4.500 amps.

. Uranium counts tend to increase with time at the optimum current value....too high and you may lose it. A good rule of thumb is to watch for the 234 counts to approach $25-100$ cps (depending on the samples). When you note these kind of counts on several passes of mass scans proceed to next step.

c. Press "Exit Program".

d. Press "Return Main Keys".

e. Press key "MS Control".

f. Press key "Input Channel”.

g. Press the number "1" key and hit enter. 
h. Press key "Input mass".

i. Type in " 235 " and hit enter.

j. Note the present count rate

k. Press key "Peak Center" (after the machine centers the beam, the printer will register a peak center value. This value should be $512 \pm$. 8.0) and repeat if necessary to obtain a good center (figure 10).

1. Repeat steps for 233 and 234. (These steps are to insure that you are getting satisfactory count rates before proceeding to begin actual analysis)

\section{Set up Analysis Table}

a. Press key "Return Main Keys".

b. Press key "ACQ”.

c. Press key "Table Set up”.

d. Press key "Analysis Table" (Table appears on screen. Edit as needed.

For “ACQ Experiment" type in "20" for Uranium metal run. For "AQ Element" type in "U3").

e. Press key "Save Table".

f. Press key "Edit end".

g. Press key "Return ACQ".

\section{Begin acquisition:}



a. Press key "Single ACQ”.
b. Press "N" when queried about starting at top of page.
c. Press Enter. This starts acquisition and print-out of results. Continue acquisition of data until desired precision is obtained.

\section{Terminate run:}

a. Press key "Abort ACQ" (not necessary if experiment has completed all the block runs).

b. Turn off filament current by simultaneously depressing the two buttons "High Speed" on the filament control box.

c. Turn KV knob down to minimum. ( 3.5-4.0)

d. Turn off "Ion-Source"

e. Tightly close the "Beam Valve" on source chamber.

f. Turn off computer monitor but not the computer itself.

TIMS procedure for Thorium metal...."Single Filament Method"

\section{Start up AM:}

a. Add liquid nitrogen to carousel. Confirm that vacuum is at least $10^{-8}$ mbar. 
b. Turn on "ion-source" by pushing button (figure 2). Turn high voltage (KV) from 4 to 10 (figure 3). Determine if multimeter display shows 9.8 -10 KV(figure 4). If it doesn't, press "Return to Main Keys", press "MS Control", press "Manual Mode", it will then read "Computer Mode", then press "Computer Mode" again. Verify that high voltage is now $\sim 9.6-10 \mathrm{KV}$..

c. Begin filament preheating when new samples have been loaded otherwise proceed to Section 3.

2. Begin filament preheating (this ensures the samples are outgassed and any organics and interfering elements are evaporated)

a. Turn KV selection knob to lowest setting (figure 3) and verify that the beam valve is closed.(figure 5).

b. Crank sample \#1 into "A” position. (figure 6)

c. Set switch on current control box to position "5". (figure 7)

d. Turn current to 3.50 amps and leave for 10 minutes.

e. Turn off current by simultaneously depressing both High Speed buttons on the filament current box.

f. Repeat steps b-e for all samples on carousel.

\section{Set Cup Configuration}

a. Press key "Return to Main keys". 

b. Press key "MS Setup”.
c. Press key "Cup Configuration”.
d. Select Th.
e. Press key “Save Conftab”.

4. Calibration Procedure (Ensures that short term changes in the high voltage are corrected for by adjusting the magnetic field to minimize system drift across the mass range)

a. Insure calibration sample (ie; U-500) is in "IS" position \#1 (figure 6).

b. Open beam valve (figure 5).

c. Turn high voltage (KV) from 4 to 10 (figure 3 ).

d. Press key "Return Main Keys".

e. Press key "MS Control".

f. Press key "Input Channel”.

g. Press the number "1" key (this is the ICM counter) and press enter..

h. Press key "Input Mass”.

i. Type in " 238 " and press enter.

j. Begin filament heating of U-500 sample.

k. Set switch on current control box to position "1". (figure 7)

1. Turn up current to 3.500 amps. Note the counts for " 238 ".

$\mathrm{m}$. When the count rate is several hundred, focus the beam. (see section 6 steps a-k) 
n. After getting a good focus and strong count rate (several thousand cps), press key "Input Mass".

o. Type in " 235 " then do a peak center and note the count rate. It should be virtually identical to the last " 238 " count (U-500 is a 1:1 ratio of $238 / 235$ ). If it is, then the machine is counting correctly and you may proceed to calibrating the DAC table.

p. Press key "Input Mass".

q. Type in " 238 " and enter

r. Press key "Input Channel”.

s. Press key "6" (this places the 238 in the \#6 Faraday cup).

t. Press key "Return Main Keys"

u. Press key "MS Calib”.

v. Press key "Magnet Calib".

w. Press key “Calib RefMass”.

x. Type "238" and enter (Screen will then indicate the current ref mass in faraday \# 6).

y. Press the upper far left button on the Ops. Control panel (figures $3 \& 8$ ).

z. Turn on the chart recorder.

aa. Adjust the three magnet dials till the digital display reads in the " 238 " range and then fine tune for the strongest signal on the chart recorder (peaking to the left). 
bb. Hit the space bar. The control will now shift back to the computer and the software will adjust the DAC tables and printout the results. All values should be close to 0.0 and must not exceed \pm 8.0 (figure 11). (If values exceed \pm 8.0 repeat steps $w$ through bb using 235 and 238 alternately and recheck till values are satisfactory).

5. Begin filament heating_(Ensure all samples have been pre-heated, the first sample to be analyzed is in the "IS" position and the beam valve is closed...figures $5 \& 6$ ).

a. Press key "Return to Main Keys".

b. Press key "Support Programs".

c. Press key "Heat/Ion/Eva" .

d. Press key "Autoheat Eva".

e. Type " 4500,100 " and hit enter. Ensure current is increasing on filament control box. This will take 45 minutes.

f. When auto-heating is complete, open the beam valve and press "Return Main Keys" (figure 5).

g. Check the pyrometer reading and note the temperature while focused on the sample location of the filament. The best ionization efficiency is attained when the temperature is between 1750 to $1900{ }^{\circ} \mathrm{C}$. Thorium is thermally sensitive. Exceeding $1900{ }^{\circ} \mathrm{C}$ will decrease ionization efficiency and rapidly deplete the sample. 
h. Press key "MS Control".

i. Press key "Input Channel”.

j. Press the number " 1 " key and enter (this is the ICM counter).

k. Press key “Input Mass”.

1. Type in "232" and hit enter.

$\mathrm{m}$. Note the present count rate. (Depending on the sample, it may be easier to acquire counts on 238 first and then return to 232).

n. Press "Peak Center" (after the machine centers the beam, the printer will register a peak center value. This value should be $512 \pm$. 8.0) and repeat if necessary to obtain a good center (figure 10).

\section{Focusing the Beam}

a. Turn on the graphing printer and remove the pen cover.

b. The printer will register the counts of the current input mass.

c. While watching the pen movements, SLOWLY adjust the barrel focus (the knob you used to put the sample filament into position...figure 6) in the direction necessary to cause the pen to move to the left (increasing). NOTE: Do not crank the knob so much as to move the filament off the electrical connection (as indicated by the sudden disappearance of current on the filament control box).....this causes the filament to quickly begin to cool down and when you return the current 
by correcting your error, the sudden zap of current may cause the sample to "pop" off the filament.

d. After getting the best count rate from the barrel focus, adjust the "shielding plate" focus knob. (figure 5)

e. Adjust the "z-deflection plate" focus (figure 11)

f. Adjust the "lens focus plates" (figure 11)

g. Press key "Peak center" (after the machine centers the beam, the printer will register a peak center value. This value should be $512 \pm$. 8.0) and repeat if necessary to obtain a good center (figure 10).

h. You should now see a marked increase in the ion count rate on the screen.

i. Repeats steps $6 \mathrm{c}$. through $6 \mathrm{~g}$ to obtain optimum focus.

j. Turn off graphing printer and place cap back on pen tip.

k. Press key "Return Main Keys"

1. Press key "Support Programs"

m. Press key "Mass scan". (A warning will come on screen....ignore it and press any key)

n. Press key "Single Icm scan”.

o. Input masses (ie. 228.5 and 232.5).

p. Press key "End Edit" to begin scan (to repeat scan...press "New Scan" and repeat steps n-p).

\section{Optimize counting conditions.}


a. Slowly increase EVA filament current. At this point you should be in the neighborhood of around 4.500 - 5.300 Amps.

b. Repeat Mass scan several times if necessary and note if all peaks are present. (ie; 229, 230 and 232) and are centered.

NOTE: Take your time!!! ........Increase filament current by 0.025 amps at a time and check the temperature of the filament. The trick is to SNEAK up on the correct temperature so as to ensure that you do not burn the sample off the filament. Ideal filament current can be as little as 4.300 amps all the way to 5.700 amps. Thorium counts tend to increase with time at the optimum current value....too high and you may lose it. A good rule of thumb is to watch for the 230 counts to approach $10-100 \mathrm{cps}$ (depending on the samples). When you note these kind of counts proceed to next step.

c. Press "Exit Program".

d. Press "Return Main Keys".

e. Press key "MS Control".

f. Press key "Input Channel”.

g. Press the number "1" key and hit enter.

h. Press key "Input mass".

i. Type in "232" and hit enter. 
j. Note the present count rate

k. Press key "Peak Center" (after the machine centers the beam, the printer will register a peak center value. This value should be $512 \pm$. 8.0) and repeat if necessary to obtain a good center (figure 10).

1. Repeat steps for 229 and 230. (These steps are to insure that you are getting satisfactory count rates before proceeding to begin actual analysis)

\section{Set up Analysis Table}

a. Press key "Return Main Keys".

b. Press key “ACQ”.

c. Press key “Table Set up”.

d. Press key "Analysis Table" (Table appears on screen. Edit as needed.

For “ACQ Experiment” type in “15” for Thorium metal run. For "AQ

Element" type in “Th").

e. Press key "Save Table”.

f. Press key "Edit end".

g. Press key "Return ACQ”.

\section{Begin acquisition:}

a. Press key "Single ACQ".

b. Press "N" when queried about starting at top of page. 
c. Press Enter. This starts acquisition and print-out of results. Continue acquisition of data until desired precision is obtained.

\section{Terminate run:}

a. Press key "Abort ACQ" (not necessary if experiment has completed all the block runs).

b. Turn off filament current by simultaneously depressing the two buttons "High Speed" on the filament control box.

c. Turn KV knob down to minimum. ( 3.5-4.0)

d. Turn off "Ion-Source"

e. Tightly close the "Beam Valve" on source chamber.

f. Turn off computer monitor but not the computer itself. 


\section{SOME MISCELLANEOUS TIPS AND TRICKS}

1. A sudden drop in the count rate may indicate that the sample is no longer at optimum focus. Interrupt the acquisition and refocus the beam.

2. During acquisition a large 2 SE (M) [\%] error during the first few blocks may indicate that the machine is not calibrated, a peak or peaks are not centered or the beam is out of focus. Make the necessary corrections and begin a new acquisition.

3. If the data appears to be in error or erratic (e.g., inconsistent data) then STOP and examine the entire system.

a. Verify that the vacuum still in the $10^{-8}$ mbar range.

b. Verify that the high voltage is still in the $10 \mathrm{KV}$ range.

c. Verify the ion counter is on.

d. Verify the beam valve is open.

e. Verify that the computer and the control panel indicators agree (e.g., mass input numbers agree with each other). If not, then reboot the software and try again (see 4 for re-booting procedure).

f. Verify that the beam is focused.

g. Verify that the peaks are centered.

h. Verify that the calibration is within tolerance.

i. Verify that the extraction lens was cleaned prior to inserting the carousel.

j. Verify that the switch on the current control box in the number " 1 " position.

4. If the computer "locks up", turn off ion counter by pressing the button on the control panel (figure 9) then turn it back on and reboot the software. When the DOS prompt appears...type mat262 and enter. Sometimes it may be necessary to cycle the electronics off and then back on along with the ion counter in order to reestablish communications between the hardware and the software. 
5. If an Uranium run is not satisfactory, it can sometimes be recovered by running the Thorium separate at a lower temperature for Uranium prior to running the Thorium. 


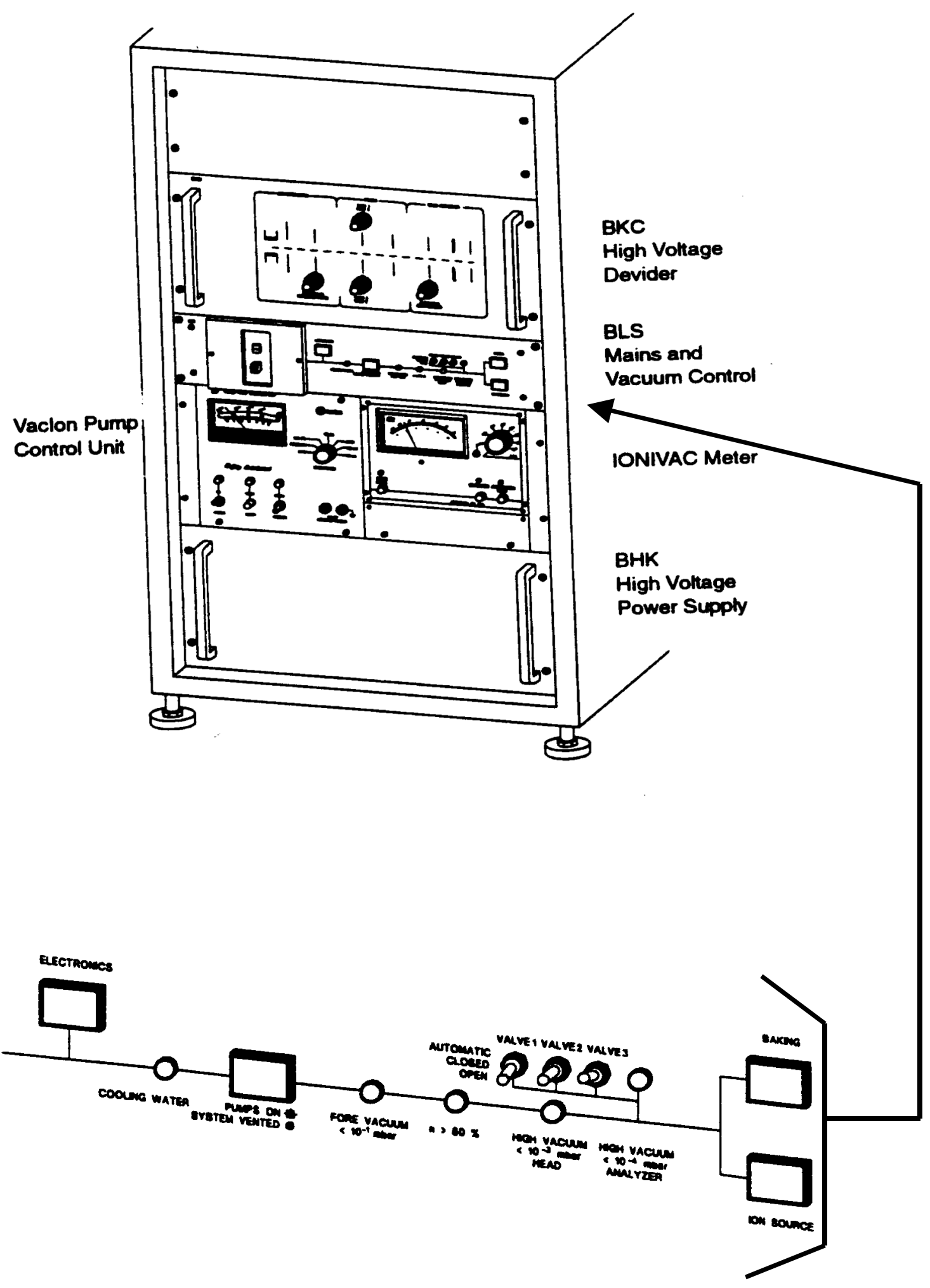

Figure 2

Close-up of control panel 


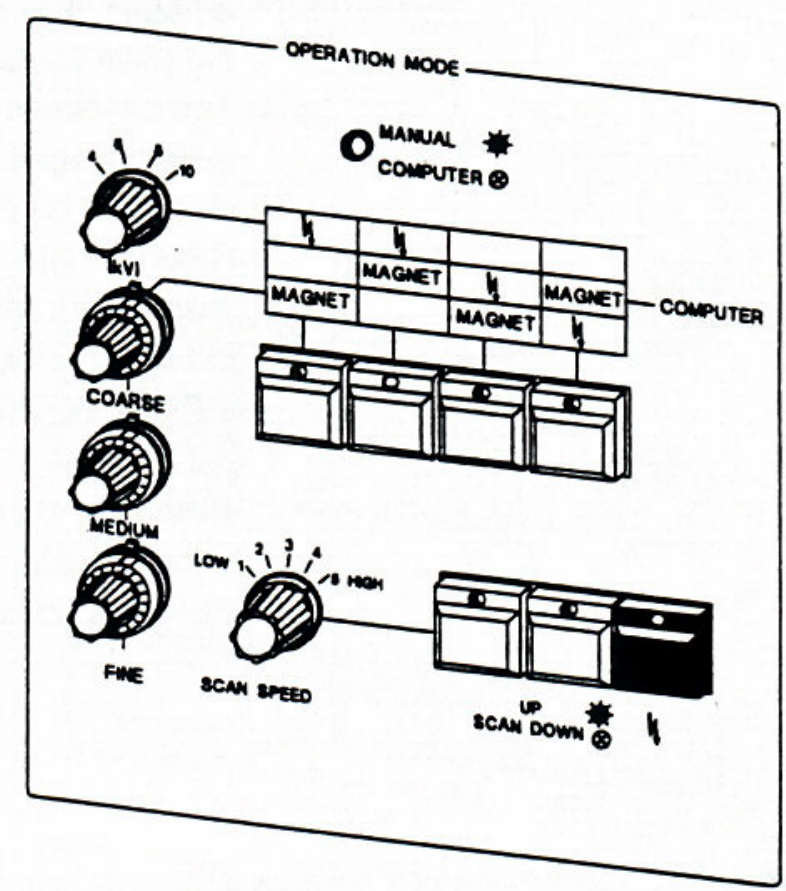

Figure 3

Operation Mode panel (on Analyzer Control unit)

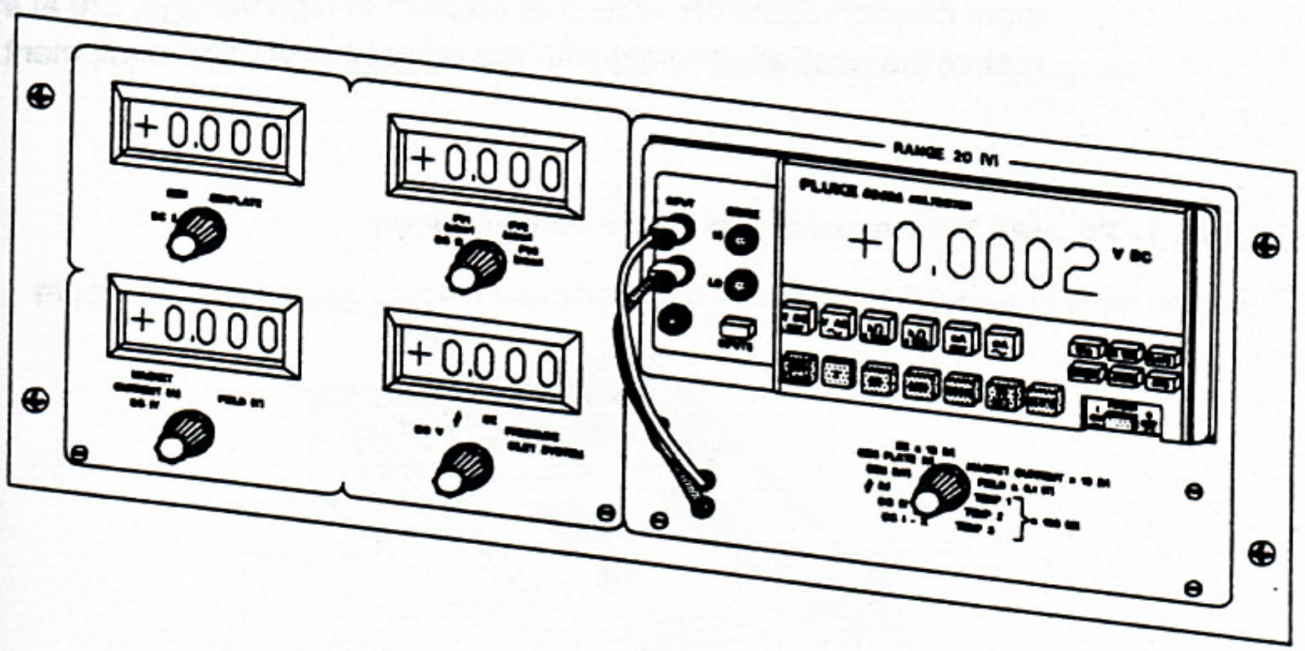

Figure 4

System Monitor with digital displays 


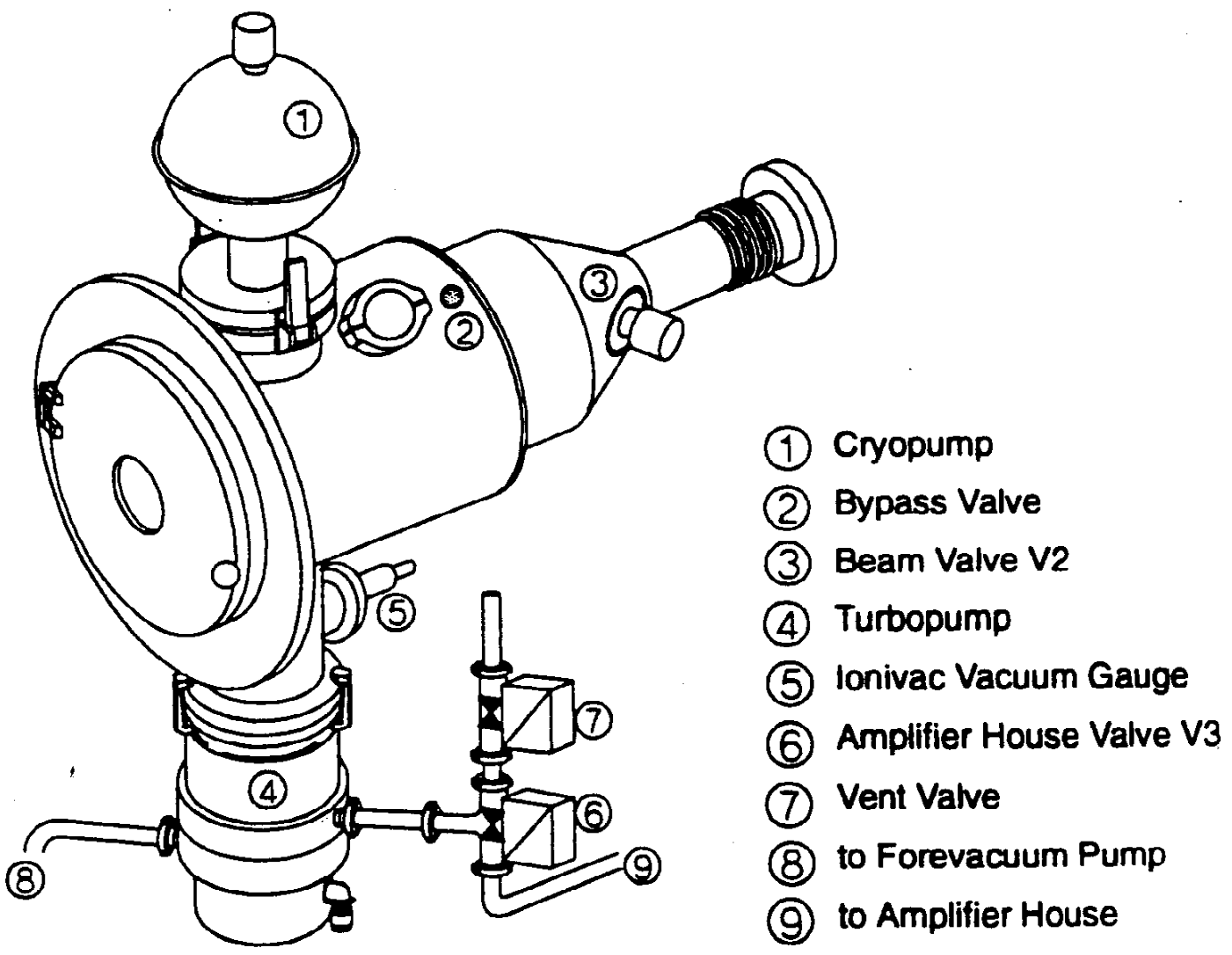

Figure 5

MAT 262 analyzer head showing the position of valves and other components 


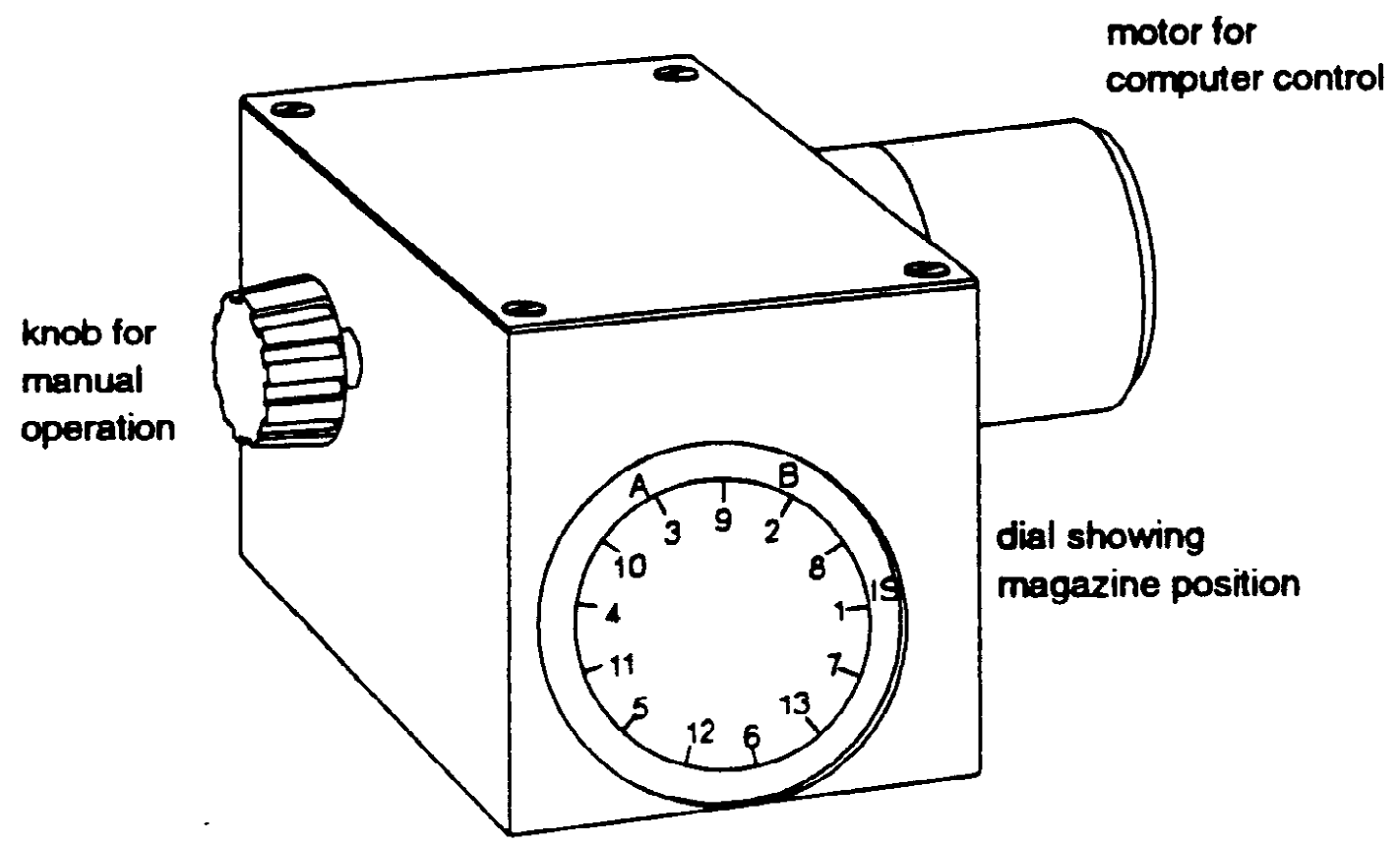

Figure 6

Magazine control unit

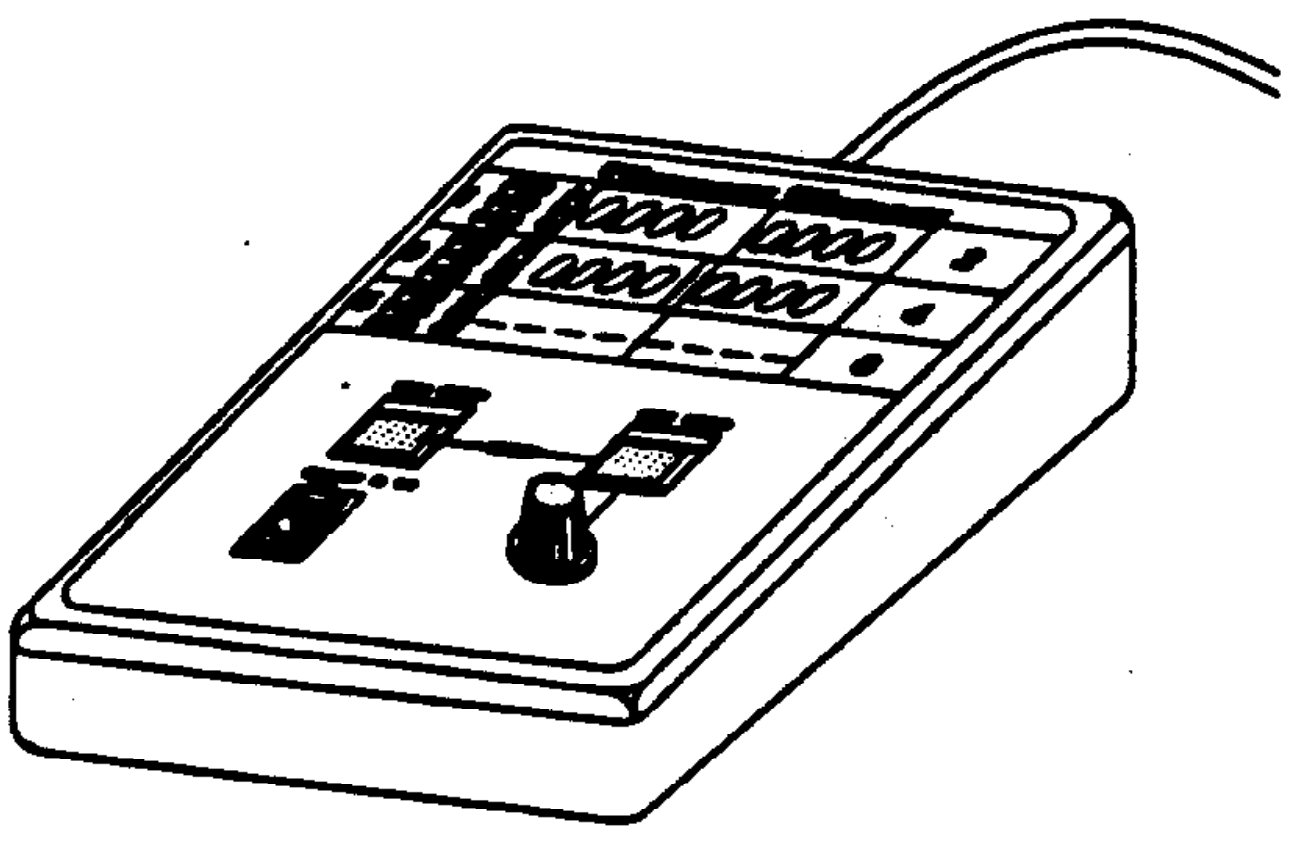

Figure 7 
Manual control for filament current

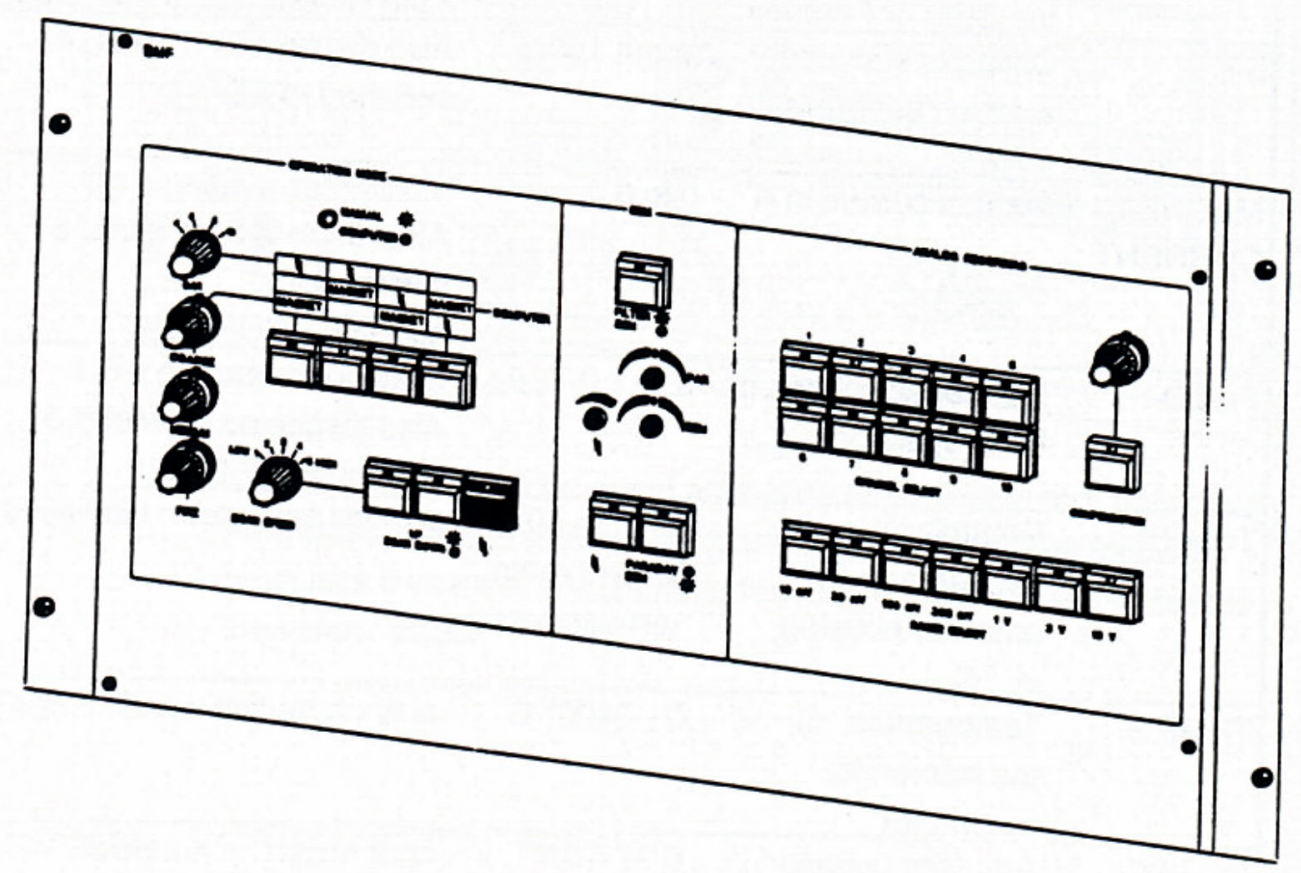

Figure 8

Analyzer Control Unit

\begin{tabular}{|c|c|c|c|c|c|c|}
\hline Peaks & an & $i$ & $\operatorname{las} 5$ & $H U-D A$ & $45 \%$ & \\
\hline MOAC & fu & tion & - MDAC & refmasstable & I** centered & $\operatorname{mas} 1$ \\
\hline MASS & $*$ & 23 & $\Rightarrow$ & 0.00 & & \\
\hline MASS & $:$ & 39 & $\approx$ & -.01 & & \\
\hline MASS & ; & 88 & $=$ & -.01 & & \\
\hline MASG & $:$ & 235 & $=$ & 0.00 & & \\
\hline MASS & $:$ & 238 & $=$ & $0.00 * *$ & 35224.373046 & \\
\hline
\end{tabular}

Figure 9

Example of HDAC printout produced following Calibration 


\begin{tabular}{|c|c|c|c|c|c|}
\hline oeakconter & : & Masa & 238 & $H V-O A C:$ & 550 \\
\hline Peakcenter & $\$$ & Mass & 238 & HU-DAC: & \\
\hline Peak & 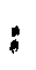 & Mass & 25 & & \\
\hline Peakcenter & $:$ & 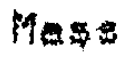 & 23 & $H W-D A C:$ & \\
\hline Pakcenter & ; & Mas & 235 & HV-DAC: & \\
\hline Peakcenter & 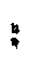 & Mass & 27 & HU-DAC: & \\
\hline Poakgenter & 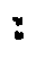 & Mo5 & 23 & $H U-D A C:$ & \\
\hline Peakcenter & & Mas & 233 & $H V-D A C$ & \\
\hline
\end{tabular}

\section{Figure 10}

Example of Printout produced following Peak Centering 


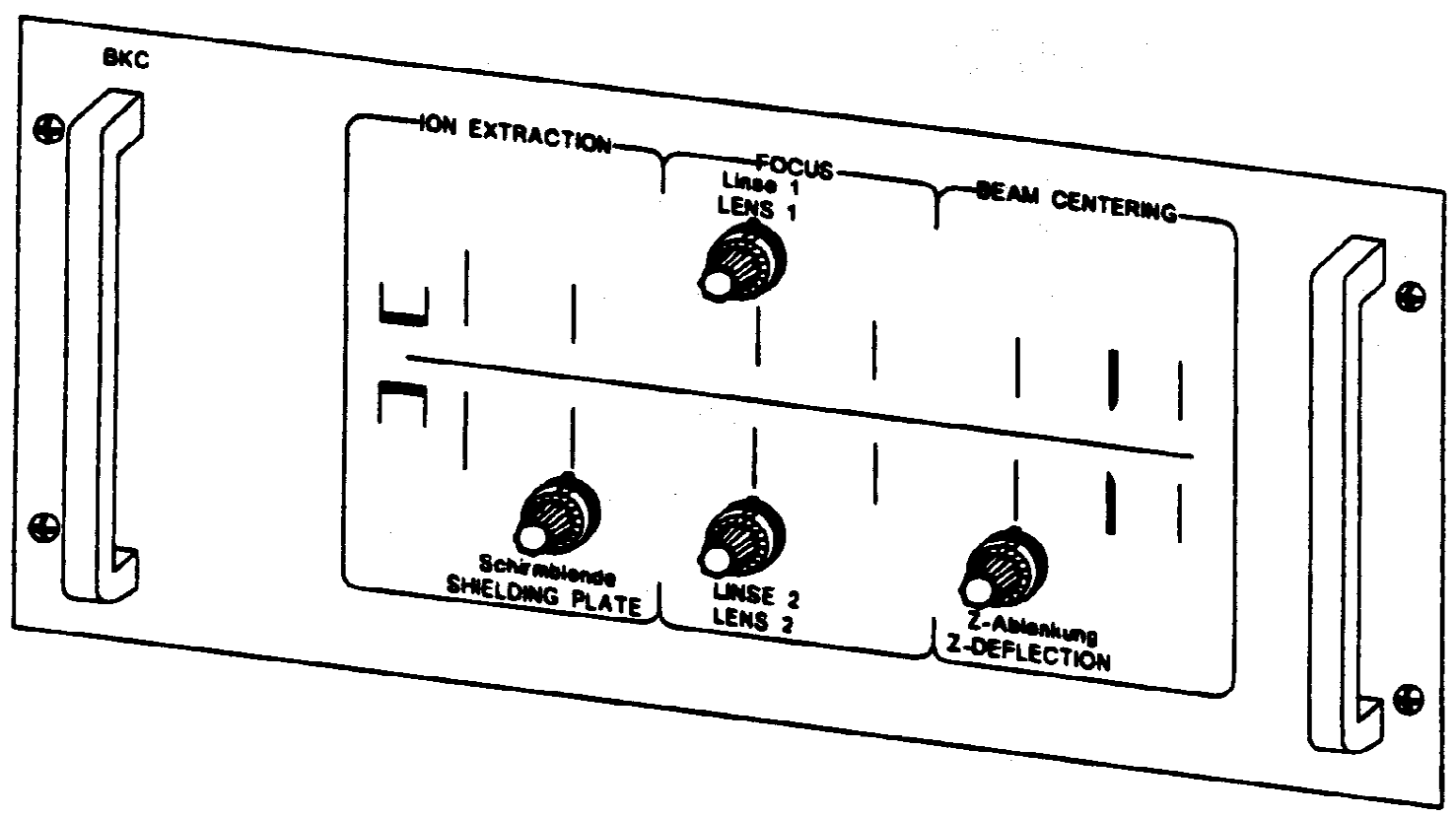

Figure 11

High Voltage Divider for Focusing 